\title{
Mucins in neoplastic spectrum of colorectal polyps: can they provide predictions?
}

\author{
Mahsa Molaei ${ }^{*}$, Babak Khoshkrood Mansoori, Reza Mashayekhi ${ }^{\dagger}$, Mohsen Vahedi $^{\dagger}$, \\ Mohamad Amin Pourhoseingholi ${ }^{\dagger}$, Seyed Reza Fatemi ${ }^{\dagger}$, Mohammad Reza Zali ${ }^{\dagger}$
}

\begin{abstract}
Background: The significance of expression of different mucins in succession of malignant transformation of colorectal polyps is not determined yet. The aim of the present study was to determine the pattern of expression of MUC1, MUC2, MUC5AC and MUC6 in colorectal polyps and to evaluate the applicability of using mucin expression in predicting the extent of malignant transformation in colorectal polyps.

Methods: A total of 454 polyp specimens comprising 36 hyperplastic polyps, 15 serrated adenomas, 258 tubular adenomas, 114 tubulovillous adenomas, and 31 villous adenomas were included in this study, and were immunostained for MUC1, MUC2, MUC5AC and MUC6 by using mucin specific antibodies.

Results: MUC1 and MUC6 were absent in all hyperplastic polyps and their expression was higher in serrated and traditional adenomas. Only 5 cases including 2 serrated adenomas, 1 tubulovillous adenoma, and 2 villous adenomas stained negative for MUC2. The highest expression of MUC5AC was observed in serrated adenomas followed by tubular adenomas. Binary logistic regression analysis indicated that positive staining for MUC1, and MUC6, and negative staining for MUC2 would increase the risk of invasion to mucosa or the muscularis mucosae in colorectal polyps. Ordinal regression analysis demonstrated a positive association between the level of staining for MUC1 and risk of being of high configuration/grade in colorectal polyps.
\end{abstract}

Conclusions: MUC1, MUC2, MUC5AC, and MUC6 have the potential to be used as predictors of malignant transformation and invasion to mucosa or the muscularis mucosae in colorectal polyps. The most reliable predictions can be achieved by determining the level of expression of MUC1.

\section{Background}

Mucins are high molecular weight glycoproteins that are produced, stored and secreted by mucosal epithelial cells [1], and act as the main part of the mucus protecting layer of the gastrointestinal tract. The protective role of mucins is achieved by the presence of oxygen bonds that attach the long sugar side-chains of the mucins to tandem repeat protein structures that are rich with serine and threonine amino acids [2]. These bonds hinder the mucin degradation by inhibition of protease activity, and so preserve the viscosity and density of mucins [2].

Various mucin proteins are encoded by different MUC genes [3-9]. MUC1 gene is located on chromosome 1q21-24 and codes for a transmembrane mucin,

\footnotetext{
* Correspondence: m_molaei@sbmu.ac.ir

† Contributed equally

Research Institute for Gastroenterology and Liver Diseases, Taleghani

Hospital, Shahid Beheshti University, M.C., Tehran, Iran
}

which is rarely expressed in normal colorectal mucosa [3]. MUC2 gene, which is aboundantly expressed in colorectal goblet cells, encodes a secretory glycoprotein [4]. The secretory MUC3 gene product is normally expressed in colorectal mucosa [4,5]. MUC4 gene encodes a transmembrane glycoprotein. This gene is located on chromosome 3q29 and predominates in intestinal mucosa [6]. MUC5AC gene forms a cluster on chromosome $11 \mathrm{p} 15.5$ with MUC2 gene, and codes for a secretory mucin in stomach [7]. Similarly, MUC6 gene is typically expressed in stomach [5].

A growing body of evidence advocates the idea that expression of MUC genes and distribution of their products alter dramatically in certain types of colorectal polyps and neoplasms $[2,10]$. These alterations take place through several mechanisms that include aberrant glycosylation of mucin side chains, immunoreactivity of mucin core peptide, deletion of normally expressed antigens, 
expression of blood-group incompatible antigens, and de novo appearance of new antigens [2]. The secretion of MUC2 gene products, for example, is suppressed in nonmucinous colorectal neoplasms $[4,11]$, or MUC5AC and MUC6, which are normally absent in colorerectal mucosa, are expressed de novo in villous and tubulovillous colorectal adenomas $[12,13]$. MUC1 is also believed to become upregulated in colorectal cancers (3).

The current consensus classifies majority of the colorectal polypoid lesions into three major levels of malignant transformation: hyperplastic polyps (HPPs), serrated adenomas, and conventional (traditional) adenomas [14-16]. It is not still elucidated, however, which mucin alterations at which stage of malignant transformation take place or become detectable [17]. For instance, there is a large body of controversy regarding the alterations of MUC5AC and MUC6 in colorectal polyps. While these two mucins were reported previously to be more expressed in medium size/stage adenomas [12], more recent data reports MUC5AC to be involved in early stages of malignant transformation, and MUC6 in final stages [18].

The main objective of this study was to determine the extent of expression of MUC1, MUC2, MUC5AC, and MUC6 in different levels of neoplastic transformation of colorectal mucosa according to immunohistochemistry results. Moreover, we aimed at determining the predictive value of mucin expression on the malignant transformation of colorectal polyps.

\section{Methods \\ Patients}

In the present study, polyp specimens of all cases of colorectal polyp, who had undergone colonoscopy and biopsy from 2005 to 2008, were retrospectively studied. Clinical and demographic data were retrieved from the available hospital records of Taleghani Hospital, Shahid Beheshti University, M.C. Colorectal polyps were classified according to their location as right sided (proximal to splenic flexure), left sided (distal to splenic flexure), and rectal.

Hematoxylin and Eosin (H\&E) slides and Formalinfixed, paraffin-embedded tissue polyp specimens including 36 hyperplastic polyps, 15 serrated adenomas, 258 tubular adenomas, 114 tubulovillous adenomas, and 31 villous adenomas were obtained from the archives of pathology department of Taleghani Hospital, Shahid Beheshti University, M.C. This classification of polyps will be referred to as polyp configuration hereafter.

Available H\&E slides were examined initially by two pathologists to recheck the original histological diagnosis of specimens. Traditional adenomas were classified in advance into three categories of mixed adenomatoushyperplastic, Low-grade adenomatous, and High-grade adenomatous polyps according to grade of glandular dysplasia.

\section{Immunohistochemistry}

Tissue samples were sectioned $(4 \mu \mathrm{m})$, deparaffinized in xylene, and rehydrated in descending alcohol gradient. Blocking solution was used to block the endogenous peroxidase activity. For antigen retrieval, sections were treated in advance in boiling citrate buffer ( $\mathrm{ph}$ 6.0) in microwave oven. Sections were immunostained and incubated afterwards with primary antibodies for MUC1 (Invitrogen, Clone:VU-4-H5), MUC2 (Invitrogen, Clone: CCP58), MUC5AC (Invitrogen, Clone:45M1), and MUC6 (Invitrogen, Clone:1G8). Then, slides were treated with Envision (DAKO, REAL Envision) for 20 minutes. To visualize immunoreactivity, diaminobenzidine was used and samples were counterstained with hematoxylin. Ascending alcohol gradient was used eventually to dehydrate the sections.

Specimens were observed by two pathologists. The level of staining for each mucin was calculated by determining the percentage of immunostained cells per high power field, and reported as 0 (no staining), $1+$ (up to $30 \%), 2+(30-60 \%$ of cells stained), $3+$ (more than $60 \%$ of cells stained). Clinical and demographic data were compared according to presence (positiveness) or absence (negativity) of immunohistochemical staining for each mucin.

\section{Statistical analysis}

Differences of distribution between the categorical variables were examined with chi-square test and Fisher's exact test in case of need. For quantitative variables Student's t-test was employed. Three factors of invasion status (to mucosa or muscularis mucosae and not beyond), configuration, and grading of polyps were considered as indicators of malignant transformation of polyps. Binary logistic regression analysis, with covariates of gender, age, polyp site, and MUC status (+/-) included, was performed to estimate the risk of invasion (as defined earlier) according to mentioned factors. In case of polyp configuration and grading, ordinal regression analysis was employed, with factors of expression level of different mucins, tumor site, and gender included, in order to generate predictions and evaluate the importance of mentioned predictor variables. Age was not included in this model because as a continous variable it would decrease the reliability of the model. Reported P values of less than 0.05 were considered to represent the statistical significance.

\section{Ethical considerations}

The present study was undertaken with approval and under direct supervision of ethics committee of 
Research Institute for Gastroenterology and Liver Diseases, affiliated to Shahid Beheshti University, M.C.

\section{Results}

The study population comprised 454 patients with mean age of $59.5 \pm 14.61$ (range 15-89), and male to female ratio of $1.48 .34 \%$ of polyps were of right colon origin, $50 \%$ of left colon, and $16 \%$ of rectum. 36 polyps $(7.9 \%)$ were hypersplastic, 15 (3.3\%) serrated adenoma, and 403 (88.8\%) traditional adenoma. Traditional adenomas included 258 tubular adenoma specimens, 114 tubulovillous adenomas, and 31 villous adenomas. Invasion (just to mucosa and muscularis mucosae) was observed only in traditional adenomas, especially the villous type (Table 1).

\section{IHC results for MUC1, MUC2, MUC5AC, and MUC6}

MUC1 was positive in 106 (23.3\%) polyps, of which none was hyperplastic. The expression of MUC1 was significantly higher in traditional adenomas than serrated adenomas/hyperplastic polyps ( $23.5 \%$ vs. $7.8 \%, \mathrm{P}=0.005)$. The main difference between the MUC1 positive and negative polyps was in the level of neoplastic transformation (Table 2). Higher levels of MUC1 expression was also observed among traditional adenomas especially tubulovillous and villous adenomas (Table 3). Immunostaining for MUC2 was positive in 449 cases (98.9\%). Only five cases had negative MUC2 staining, of which 2 were serrated adenomas, 1 was tubulovillous adenoma, and 2 were villous adenomas (Table 2). In comparison to hyperplastic polyps, traditional adenomas showed lower levels of MUC2 expression (Table 3).

MUC5AC was positive in 195 specimens. We could find no significant difference in distribution of MUC5AC in different polyps. In general, majority of colorectal polyps in this study had negative expression of MUC5AC. The exception, however, were serrated adenomas of which $66 \%$ were MUC5AC positive (Table 2). More than $50 \%$ of serrated adenomas had MUC5AC expression level of $2+$ or more (Table 3 ). In addition, meanwhile MUC6 was positive in about one third of tubulovillous adenoma and villous adenomas, it was negative in all hyperplastic polyps (Table 2). Nonetheless, no statistical significant could be found for difference of MUC6 expression level in various polyp types (Table 3). Moreover, we could find no association between expression of MUC6 with MUC5AC; in other words, only $24.1 \%$ of MUC5AC positive specimens were also positive more MUC6, and 47\% of MUC6 positive specimens, had also positive immunostaining for MUC5AC ( $\mathrm{P}=0.354)$.

Certain patterns of MUC expression were significantly associated with invasion of polyps to mucosa or muscularis mucosae, including the up-regulation of MUC1 (marginal significance), absence of MUC2, and positive MUC6 expression (Table 2). Regression analysis, with covariates of gender, age, polyp site, and MUC status included, indicated that negative staining for MUC2, Positive staining for MUC6, and left-sidedness would increase the risk of mucosal or muscularis mucosae invasion in colorectal polyps significantly (Table 4).

The ordinal regression analysis indicated that the described model (see statistical analysis), would give adequate predictions either in case of configuration and grading $(\mathrm{P}<0.001$, Chi square $=65.32$ and 64.99 respectively). The model with the complementary log-log link (table 5) shows that the four thresholds of the model equation were significantly different from zero and substantially contributed to the values of the response probability in different configurations. Moreover, the configuration of polyps was significantly associated with three covariates: polyp site, and level of expression of MUC1 and MUC5AC (table 5).

Using the model with the negative log-log link to build the ordinal regression, the grade of glandular dysplasia was found to be significantly associated with the three variables of expression levels of MUC1 and MUC2, and polyp site (table 6).

\section{Discussion}

MUC1 and MUC2 normally belong to colorectal mucosa; however, while the expression of MUC1 is a rare occasion

Table 1 invasion ${ }^{1}$ status, grading, and site of traditional adenomas

\begin{tabular}{|c|c|c|c|c|}
\hline & $\mathrm{TA}^{2}$ & TVA $^{2}$ & $\mathrm{VA}^{2}$ & $P$ value \\
\hline Invasion & $3(1.2 \%)$ & $10(8.8 \%)$ & $11(35.5 \%)$ & $<0.0001$ \\
\hline Grade & & & & $<0.0001$ \\
\hline Mixed adenomatous-hyperplastic & $17(6.6 \%)$ & $0 \%$ & $0 \%$ & \\
\hline Low-grade adenomatous & $217(84.1 \%)$ & $76(66.7 \%)$ & $16(51.6 \%)$ & \\
\hline High-grade adenomatous & $24(9.3 \%)$ & $38(33.3 \%)$ & $15(48.4 \%)$ & \\
\hline Polyp Site & & & & $<0.0001$ \\
\hline Right colon & $103(39.9 \%)$ & $27(23.7 \%)$ & $5(16.1 \%)$ & \\
\hline Left colon & $118(45.7 \%)$ & $64(56.1 \%)$ & $21(67.7 \%)$ & \\
\hline Rectum & $37(14.3 \%)$ & $22(19.3 \%)$ & $5(16.1 \%)$ & \\
\hline
\end{tabular}

${ }^{1}$ Invasion not beyond mucosa or muscularis mucosae; ${ }^{2}$ Abbreviations: TA, tubular adenoma; TVA, tubulovillous adenoma; VA, villous adenoma. 
Table 2 Clinical and demographic differences of polyps with and without MUC immunoreactivity

\begin{tabular}{|c|c|c|c|c|c|c|c|c|}
\hline & \multicolumn{2}{|c|}{ MUC1 Staining } & \multicolumn{2}{|c|}{ MUC2 Staining } & \multicolumn{2}{|c|}{ MUC5AC Staining } & \multicolumn{2}{|c|}{ MUC6 Staining } \\
\hline & + & - & + & - & + & - & + & - \\
\hline \multicolumn{9}{|l|}{$\overline{\text { Age }}$} \\
\hline \multirow[t]{2}{*}{ Mean } & 59.86 & 59.45 & 59.55 & 59.00 & 59.37 & 59.67 & 61.14 & 59.10 \\
\hline & \multicolumn{2}{|c|}{$P=0.803$} & \multicolumn{2}{|c|}{$P=0.933$} & \multicolumn{2}{|c|}{$P=0.837$} & \multicolumn{2}{|c|}{$P=0.231$} \\
\hline \multicolumn{9}{|l|}{ Gender } \\
\hline Male & 72 & 198 & 266 & 4 & 112 & 158 & 61 & 209 \\
\hline \multirow[t]{2}{*}{ Female } & 33 & 150 & 182 & 1 & 83 & 100 & 39 & 144 \\
\hline & \multicolumn{2}{|c|}{$P=0.020$} & \multicolumn{2}{|c|}{$P=0.462$} & \multicolumn{2}{|c|}{$P=0.491$} & \multicolumn{2}{|c|}{$P=0.824$} \\
\hline \multicolumn{9}{|l|}{ Site } \\
\hline Right colon & 37 & 119 & 154 & 2 & 73 & 83 & 27 & 127 \\
\hline Left colon & 54 & 172 & 224 & 2 & 94 & 132 & 57 & 169 \\
\hline \multirow[t]{2}{*}{ Rectum } & 15 & 56 & 70 & 1 & 27 & 44 & 14 & 57 \\
\hline & \multicolumn{2}{|c|}{$P=0.908$} & \multicolumn{2}{|c|}{$P=0.975$} & \multicolumn{2}{|c|}{$P=0.370$} & \multicolumn{2}{|l|}{0.404} \\
\hline \multicolumn{9}{|l|}{ Configuration } \\
\hline Hyperplastic & 0 & 36 & 36 & 0 & 13 & 23 & 0 & 36 \\
\hline Serrated & 4 & 11 & 13 & 2 & 10 & 5 & 4 & 11 \\
\hline $\mathrm{TA}^{1}$ & 69 & 189 & 258 & 0 & 117 & 141 & 57 & 201 \\
\hline TVA $^{1}$ & 21 & 93 & 113 & 1 & 43 & 71 & 28 & 86 \\
\hline \multirow[t]{2}{*}{$V A^{1}$} & 12 & 19 & 29 & 2 & 12 & 19 & 11 & 20 \\
\hline & \multicolumn{2}{|c|}{$P=0.001$} & \multicolumn{2}{|c|}{$P<0.0001$} & \multicolumn{2}{|c|}{$P=0.182$} & \multicolumn{2}{|c|}{$P=0.007$} \\
\hline \multicolumn{9}{|l|}{ Type } \\
\hline Hyperplastic & 0 & 36 & 36 & 0 & 13 & 23 & 0 & 36 \\
\hline Serrated & 4 & 11 & 13 & 2 & 10 & 5 & 4 & 11 \\
\hline Mixed adenomatous-hyperplastic & 0 & 17 & 17 & 0 & 3 & 14 & 3 & 14 \\
\hline Low-grade adenomatous & 70 & 239 & 309 & 0 & 136 & 173 & 74 & 235 \\
\hline \multirow[t]{2}{*}{ High-grade adenomatous } & 32 & 45 & 74 & 3 & 33 & 44 & 19 & 58 \\
\hline & $p<0$ & & $p<0$ & & $P=0$ & & $P=0$. & \\
\hline Invasion $^{2}$ & $9.4 \%$ & $4 \%$ & $4.7 \%$ & $60 \%$ & $3.6 \%$ & $6.6 \%$ & $13 \%$ & $3.1 \%$ \\
\hline & $P=0$. & & $p<0$ & & $P=0$ & & $p<0$. & \\
\hline
\end{tabular}

${ }^{1}$ Abbreviations: TA, tubular adenoma; TVA, tubulovillous adenoma; VA, villous adenomas; ${ }^{2}$ Invasion not beyond mucosa or muscularis mucosae.

in normal colorectal tissue, MUC2 is the predominant secretory colorectal mucin [17-21]. MUC5AC and MUC6, which are gastric mucins, are believed to express de novo in certain colorectal polyps [12-14].

The pattern of distribution of different mucins described in this study is in general in accordance with findings of previous studies in this regard $[2,4,12,14,16-21]$. While the highest expression of MUC1, and MUC6 was observed in villous adenomas, they were totally absent in hyperplastic polyps. MUC2, in contrast, was completely expressed in hyperplastic polyps and tubular adenomas, but was absent in some serrated adenoma, tubulovillous adenoma, and villous adenoma specimens.

Results of MUC5AC, however, were in favor of previous studies depicting a higher expression of MUC5AC in middle stages of malignant transformation [12]. Positive coefficients for MUC5AC in ordinal regression analysis (table 5) indicate that increase of MUC5AC expression level does not increase the likelihood of a polyp to be of villous adenoma configuration. These results were consistent with that of table 3, in which expression of MUC5AC was more prominent in tubular adenoma, and to a lesser extent in serrated and tubulovillous adenomas.

As is shown in table 2, when it comes to the distribution pattern of different mucins, serrated adenomas act as if they rank higher in the sequence of grading and configuration. This is much more tangible in case of MUC2 that was absent in $13.3 \%$ of serrated adenomas, and MUC5AC that was present in $66.6 \%$ of these cases. These findings advocate the results reported by some previous studies regarding the high abnormality of mucins expression in serrated adenomas $[14,18]$.

Along with determining the pattern of distribution of mucins in colorectal polyps, the present study benefits from the added value of ordinal regression analysis as part of its methodology. The model fitting information tables indicated that our models would give better predictions than intercept-only models (see results) [22-24]. 
Table 3 Level of expression of different mucins in colorectal polyps

\begin{tabular}{cllllll}
\hline & HPP $^{1}$ & $\mathbf{S A}^{1}$ & $\mathbf{T A}^{1}$ & TVA $^{1}$ & $\mathbf{V A}^{\mathbf{1}}$ & $\boldsymbol{P}$ value \\
\hline MUC1 expression & & & & & & \\
0 & 36 & 11 & 189 & 93 & 19 & $<0.000$ \\
$1+$ & 0 & 3 & 58 & 13 & 2 & \\
$2+$ & 0 & 1 & 11 & 8 & 8 & \\
$3+$ & 0 & 0 & 0 & 0 & 2 & \\
MUC2 expression & & & & & & \\
0 & 0 & 2 & 0 & 1 & 2 & $<0.000$ \\
$1+$ & 3 & 2 & 32 & 16 & 2 & \\
$2+$ & 22 & 6 & 145 & 69 & 15 & \\
$3+$ & 11 & 5 & 81 & 28 & 12 & \\
MUC5AC expression & & & & & & \\
0 & 23 & 5 & 141 & 71 & 19 & $<0.000$ \\
$1+$ & 10 & 2 & 16 & 13 & 10 & \\
$2+$ & 2 & 6 & 74 & 27 & 2 & \\
$3+$ & 1 & 2 & 27 & 3 & 0 & \\
MUC6 expression & & & & & & \\
0 & 36 & 11 & 201 & 86 & 20 & 0.067 \\
$1+$ & 0 & 3 & 48 & 23 & 8 & \\
$2+$ & 0 & 1 & 8 & 5 & 2 & \\
$3+$ & 0 & 0 & 1 & 0 & 1 & \\
\hline
\end{tabular}

${ }^{1}$ Abbreviations: HPP, hyperplastic polyps; SA, serrated adenoma; TA, tubular adenoma; TVA, tubulovillous adenoma; VA, villous adenoma.

Moreover, the Pearson chi-square and deviance chisquare were non-significant for both models, implying the well fitting. As no scale component was included, test of parallel lines assumption was applicable [22], and yielded significance of less than 0.001 for both models (not presented in results section).

Given the regression analyses, (binary logistic and ordinal) it is implied that different mucins can provide certain predictions regarding mucosal and muscularis mucosae invasion, grading and configuration of colorectal polyps. In this respect, MUC 2 and 6 were shown to be associated with invasive behaviour of these polyps. Level of expression of MUC1 and 5 was associated with the level of configuration, and level of expression of MUC 1 and 2 could provide predictions on grading of colorectal polyps.
MUC1 exhibited negative regression coefficients in both of these ordinal regression models, indicating that polyps that expressed lower levels of immunostaining for MUC1 were unlikely to rank higher in polyp configuration/grading. Moreover, in binary logistic regression analysis, a marginally significant association was observed between the expression of MUC1 and invasion risk. At the present state of knowledge, these findings do not imply that MUC1 can be used as a tumor marker. But based upon these models, one can confidently argue about the potential applicability of MUC1 expression as a predictor of malignant transformation, and invasive behavior of colorectal polyps. Negative staining for MUC2 was shown in the binary logistic regression model to be valuable in predicting the risk of mucosal and muscularis mucosae invasion in colorectal polyps. In addition, in ordinal regression analysis, it was shown to reduce the likelihood of a polyp to be of high grades. Positive expression of MUC6 increased the risk of invasion (as defined earlier) significantly; however, no predictions could be elicited in terms of grading and configuration by using MUC6.

Applicability of mucins in predicting certain characteristics of colorectal polyps (invasion, configuration and grading) evokes the notion that maybe mucins expression could be employed in predicting the extent of malignant transformation in colorectal polyps. Future studies, however, would prove or disprove this hypothetical potential.

In case of polyp site, significant associations were elicited in all of regression models with invasion, grading and configuration. Given these analyses, beside the expression status of MUC1, left sidedness of colorectal polyps corresponded to increased risk of invasion to mucosa and muscularis mucosae, having high configuration and polyp grading.

Our models had their own limitations too. The major limitation, which arised from the retrospective nature of the study, was that data of normal colorectal mucosa were not included in the statistical models. The fact is that the pattern of expression of MUC1, MUC2, and

Table 4 Regression analysis determining the risk of invasion ${ }^{1}$ in colorectal polyps

\begin{tabular}{lllll}
\hline & $\boldsymbol{P}$ value & Odds Ratio & \multicolumn{2}{l}{$\mathbf{9 5 \% \text { confidence interval }}$} \\
\cline { 3 - 5 } & & & Lower bound & Upper bound \\
\hline MUC1, negative vs. positive & 0.069 & 0.407 & 0.155 & 1.071 \\
MUC2, negative vs. positive & 0.002 & 29.729 & 3.424 & 258.140 \\
MUC5AC, negative vs. positive & 0.132 & 2.142 & 0.794 & 5.776 \\
MUC6, negative vs. positive & 0.001 & 0.203 & 0.081 & 0.506 \\
Gender, female vs. male & 0.053 & 2.503 & 0.988 & 6.337 \\
Polyp site, left colon and rectum vs. right colon & 0.049 & 3.834 & 1.005 & 1.005 \\
Age $^{2}$, & 0.335 & 0.985 & 0.956 & 1.015 \\
\hline
\end{tabular}

\footnotetext{
${ }^{1}$ Invasion to mucosa or muscularis mucosae and not beyond; ${ }^{2}$ age was entered as continuous variable.
} 
Table 5 ordinal regression determining the risk of ranking higher in the configuration of colorectal polyps

\begin{tabular}{|c|c|c|c|c|}
\hline & \multirow[t]{2}{*}{$P$ value } & \multirow[t]{2}{*}{ Regression Coefficient } & \multicolumn{2}{|c|}{$95 \%$ confidence interval } \\
\hline & & & Lower bound & Upper bound \\
\hline \multicolumn{5}{|l|}{ Threshold } \\
\hline $\mathrm{HPP}^{1}$ & $<0.001$ & -20.295 & -22.239 & -18.325 \\
\hline$S A^{1}$ & $<0.001$ & -19.929 & -21.864 & -17.993 \\
\hline $\mathrm{TA}^{1}$ & $<0.001$ & -17.580 & -19.508 & -15.658 \\
\hline TVA $^{1}$ & $<0.001$ & -16.586 & -18.506 & -14.666 \\
\hline \multicolumn{5}{|l|}{ MUC1 } \\
\hline 0 & $<0.001$ & -17.609 & -18.075 & -17.142 \\
\hline $1+$ & $<0.001$ & -17.810 & -18.334 & -17.286 \\
\hline $2+$ & - & -16.873 & -16.873 & -16.873 \\
\hline \multicolumn{5}{|l|}{ MUC5AC } \\
\hline 0 & 0.008 & 0.583 & 0.152 & 1.014 \\
\hline $1+$ & 0.001 & 0.865 & 0.345 & 1.384 \\
\hline $2+$ & 0.039 & 0.480 & 0.024 & 0.937 \\
\hline \multicolumn{5}{|l|}{ Polyp site, } \\
\hline $\begin{array}{l}\text { left colon and rectum } \\
\text { right colon }\end{array}$ & $<0.001$ & 0.417 & 0.194 & 0.641 \\
\hline
\end{tabular}

${ }^{1}$ Abbreviations: HPP, hyperplastic polyps; SA, serrated adenoma; TA, tubular adenoma; TVA, tubulovillous adenoma.

MUC6 that is reported in the present article for hyperplastic polyps, is identical to what is reported in other studies for normal mucosa $[14,17,18]$. However, this does not hold true for MUC5AC, which indicates that lack of inclusion of normal mucosa data could have influenced the regression results of MUC5AC to a larger extent.

The other limitation, arised from the recent concept that serrated adenomas might go through a different pathway of malignant transformation [25-28]. Majority of colorectal polyps can be classified as hyperplastic polyps, serrated adenomas and traditional adenomas [2].
Owning mixed features of hyperplastic polyps and traditional adenomas, serrated adenomas were quite recently introduced as a distinct entity that lies between hyperplastic polyps and traditional adenomas in the sequence of malignant transformation of colorectal polyps [29]. However, more recent data indicate that serrated adenomas can get involved in an alternative carcinogenesis pathway that does not include traditional adenomas [25-28]. This could cast a shadow of doubt on the presumed ordinal hierarchy towards the malignancy that was considered in this study in terms of grading and

Table 6 ordinal regression determining the risk of being of higher grades

\begin{tabular}{|c|c|c|c|c|}
\hline & \multirow[t]{2}{*}{$P$ value } & \multirow[t]{2}{*}{ Regression Coefficient } & \multicolumn{2}{|c|}{ 95\% confidence interval } \\
\hline & & & Lower bound & Upper bound \\
\hline \multicolumn{5}{|l|}{ Threshold } \\
\hline $\mathrm{HPP}^{1}$ & $<0.001$ & -8.692 & -10.818 & -6.566 \\
\hline$S A^{1}$ & $<0.001$ & -8.515 & -10.640 & -6.391 \\
\hline Mixed adenomatous-hyperplastic & $<0.001$ & -8.352 & -10.467 & -6.228 \\
\hline Low-grade adenomatous & $<0.001$ & -5.763 & -7.855 & -3.670 \\
\hline \multicolumn{5}{|l|}{ MUC1 } \\
\hline 0 & $<0.001$ & -6.857 & -7.451 & -6.263 \\
\hline $1+$ & $<0.001$ & -6.172 & -6.807 & -5.537 \\
\hline $2+$ & - & -5.189 & -5.189 & -5.189 \\
\hline \multicolumn{5}{|l|}{ MUC2 } \\
\hline 0 & 0.043 & -1.345 & -2.651 & -0.040 \\
\hline $1+$ & 0.251 & .221 & -0.156 & 0.598 \\
\hline $2+$ & 0.682 & .052 & -0.196 & 0.299 \\
\hline \multicolumn{5}{|l|}{ Polyp site, } \\
\hline Left colon and rectum & 0.047 & 0.237 & 0.003 & 0.470 \\
\hline Right colon & & & & \\
\hline
\end{tabular}

\footnotetext{
${ }^{1}$ Abbreviations: HPP, hyperplastic polyps; SA, serrated adenoma.
} 
configuration. Nonetheless, at the present state of knowledge, we preferred to adhere to these two commonly used classifications, and not to exclude serrated adenomas.

\section{Conclusions}

To conclude, we think that mucins have the potential provide predictions on invasive behaviour, grading and configuration of colorectal polyps. But the predictive value of various mucins differ based upon what is going to be predicted. Almost all of mucins that are studied here can provide predictions on the invasive behavior of colorectal polyps; however, only MUC1 can provide such information in respect to both the grading and configuration of colorectal polyps. Thus, MUC1 is the most reliable predictor of malignant transformation of colorectal polyps among the mucins that are studies in this article.

\section{Acknowledgements}

Authors appreciate the efforts of RIGLD for funding and support.

\section{Authors' contributions}

MM participated in study design, carried out the immunostaining, and was one of the blinded pathologists who examined the slides. BKM participated in the study design, coded the slides (blinding), performed the statistical analysis, and wrote the article. RM participated in immunostaining, and was one of blinded pathologists who examined the slides. MV and MAP participated in data collection, mining, and statistical analysis. SRF participated in study design, provided data, and funding by RIGLD.MRZ participated in study design, and supervised the study. All authors read and approved the final manuscript.

\section{Competing interests}

The authors declare that they have no competing interests.

Received: 19 April 2009 Accepted: 7 October 2010

Published: 7 October 2010

\section{References}

1. Kim YS, Gum JR, Byrd JC, Toribara NW: The structure of human intestinal apomucins. Am Rev Respir Dis 1991, 144:10-14.

2. Niv Y: Mucin and Colorectal Cancer. IMAJ 2000, 2:775-777.

3. Swallow DM, Gendler S, Griffiths B, Kearney A, Povey S, Sheer D, Palmer RW, Taylor-Papadimitriou J: The hypervariable gene locus PUM, which codes for the tumor associated epithelial mucins, is located on chromosome 1, within the region 1q21-24. Ann Hum Genet 1987, 51:289-294.

4. Weiss AA, Babyatsky MW, Ogata S, Chen A, Itzkowitz SH: Expression of MUC2 and MUC3 mRNA in human normal, malignant and inflammatory tissues. J Histochem Cytochem 1996, 44:1161-1166.

5. Gendler SJ, Spicer AP: Epithelial mucin genes. Annu Rev Physiol 1995, 57:607-634.

6. Nollet S, Moniaux N, Maury J, Petitprez D, Degand P, Laine A, Porchet N, Aubert JP: Human mucin gene MUC4: organization of its $5^{\prime}$ region and polymorphism of its central tandem repeat array. Biochem J 1998, 332:739-748

7. Pigny P, Guyonnet Duperat V, Hill AS, Pratt WS, Galiegue-Zouitina S, d'Hooge MC, Laine A, Van-Seuningen I, Degand P, Gum JR, Kim YS, Swallow DM, Aubert JP, Porchet N: Human mucin genes assigned to $11 \mathrm{p} 15.5$ : identification and organization of a cluster of genes. Genomics 1996, 38:340-352.

8. Bobek LA, Tsai H, Biesbrock AR, Levine MJ: Molecular cloning, sequence and specificity of expression of the gene encoding the low molecular weight human salivary mucin (MUC7). J Biol Chem 1993, 268:20563-20569.
9. Lapensée L, Paquette Y, Bleau G: Allelic polymorphism and chromosomal localization of the human oviductin gene (MUC9). Fertil Steril 1997, 68:702-708.

10. Kim YS: Mucin glycoprotein alterations in the gastrointestinal malignancies and metastasis. Eur J Gastroenterol Hepatol 1993, 5:219-225.

11. Hanski C, Riede E, Gratchev A, Foss HD, Bohm C, Klussmann E, Hummel M, Mann B, Buhr HJ, Stein H, Kim YS, Gum J, Riecken EO: MUC2 gene suppression in human colorectal carcinomas and their me- tastases: in vitro evidence of the modulatory role of DNA methylation. Lab Invest 1997, 77:685-695.

12. Bartman AE, Sanderson SJ, Ewing SL, Niehans GA, Wiehr CL, Evans MK, Ho SB: Aberrant expression of MUC5AC and MUC6 gastric mucin genes in colorectal polyps. Int J Cancer 1999, 80:210-218.

13. Forgue-Lafitte ME, Fabiani B, Levy PP, Maurin N, Fléjou JF, Bara J: Abnormal expression of M1/MUC5AC mucin in distal colon of patients with diverticulitis, ulcerative colitis and cancer. Int J Cancer 2007, 121:1543-1549

14. Biemer-Hüttmann $A E$, Walsh MD, McGuckin MA, Ajioka $Y$, Watanabe $H$ Leggett BA, Jass JR: Immunohistochemical staining patterns of MUC1, MUC2, MUC4, and MUC5AC mucins in hyperplastic polyps, serrated adenomas, and traditional adenomas of the colorectum. J Histochem Cytochem 1999, 47:1039-1048.

15. Snover DC, Jass JR, Fenoglio-Preiser C, Batts KP: Serrated polyps of the large intestine: a morphologic and molecular review of an evolving concept. Am J Clin Pathol 2005, 124:380-391.

16. Paris MJ, Williams BRG: Characterization of a 500-kb contig spanning the region between c-Ha-Ras and MUC2 on chromosome 11p15.5. Genomics 2000, 69:196-202.

17. Aksoy N, Akinci F: Mucin macromolecules in normal, adenomatous, and carcinomatous colon: evidence for the neotransformation. Macromol Biosci 2004, 4:483-496.

18. Perçinel S, Savaș B, Ensari A, Kuzu I, Kuzu MA, Bektaș M, Cetinkaya H, Kurşun N: Mucins in the colorectal neoplastic spectrum with reference to conventional and serrated adenomas. Turk J Gastroenterol 2007, 18:230-238.

19. Reid JC, Harris A: Developmental expression of mucin genes in the human gastrointestinal system. Gut 1998, 42:220-226.

20. Audie JP, Janin A, Porchet N, Copin MC, Gosselin B, Aubert JP: Expression of human mucin genes in respiratory, digestive, and reproductive tracts ascertained by in situ hybridization. J Histochem Cytochem 1993, 41:1479-1485.

21. Ogata S, Uehara H, Itzkowits SH: Mucin gene expression in colonic tissues and cell lines. Cancer Res 1992, 52:5971-5978.

22. Bender $R$, Grouven U: Ordinal logistic regression in medical research. Journal of the Royal College of Physicians of London 1997, 31:546-551.

23. Coste J, Walter E, Wasserman D, Venot A: Optimal discriminant analysis for ordinal responses. Statistics in Medicine 1997, 16:561-569.

24. Ananth C, Kleinbaum D: Regression models for ordinal responses: a review of methods and applications. International Journal of Epidemiology 1997, 26:1323-1333.

25. Yao T, Kouzuki T, Kajiwara M, Matsui N, Oya M, Tsuneyoshi M: Serrated adenoma of the colorectum, with reference to its gastric differentiation and its malignant potential. J Pathol 1999, 187:511-517.

26. Jass JR: Serrated adenoma of the colorectum. Curr Diagn Pathol 2002, 8:42-49.

27. Jass JR: Pathogenesis of colorectal cancer. Surg Clin N Am 2002, 82:891-904.

28. Huang CS, O'Brien MJ, Yang S, Farraye FA: Hyperplastic polyps, serrated adenomas, and the serrated polyp neoplasia pathway. Am J Gastroenterol 2004, 99:2242-2255.

29. Longacre TA, Fenoglio-Preiser CM: Mixed hyperplastic adenomatous polyps: serrated adenomas: A distinct form of colorectal neoplasia. Am J Surg Pathol 1990, 14:524-537.

\section{Pre-publication history}

The pre-publication history for this paper can be accessed here: http://www.biomedcentral.com/1471-2407/10/537/prepub

doi:10.1186/1471-2407-10-537

Cite this article as: Molaei et al:: Mucins in neoplastic spectrum of colorectal polyps: can they provide predictions? BMC Cancer 2010 10:537. 